\title{
Study on the Health Application of the Snow Stepping Sound
}

\author{
Zhixing Tian", Ik-soo Ahn² and Myung-Jin Bae ${ }^{3 *}$

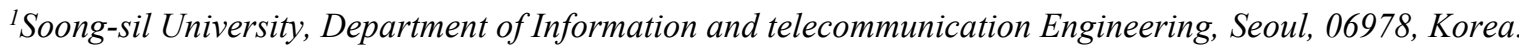 \\ Orcid Id : 0000-0003-3882-2459 \\ ${ }^{2}$ Soong-sil University, Department of Information and telecommunication Engineering, Seoul, 06978, Korea. \\ Orcid Id : 0000-0003-1144-1963 \\ ${ }^{3}$ Soong-sil University, Department of Information and telecommunication Engineering, Seoul, 06978, Korea. \\ Orcid Id : 0000-0002-7585-0400
}

\begin{abstract}
:
In recent years, ASMR audio has become popular on the Internet, and people begin to pay more attention to the spiritual feelings brought by sound. They look for various sounds to be applied in different scenes, the purpose is to activate the brain through sound stimulation [1]. In addition to man-made music, various voices in nature are also welcomed. However, there is still a lack of research on the nature of these sounds, and most of them still remain on the assessment of people's subjective feelings. The sound of treading snow is one of the most popular natural sounds. Therefore, this paper analyzes the sound of snow stepping from the perspective of signal processing, and mainly studies the time domain waveform and frequency domain energy distribution of the sound. By observing the instantaneous changes of the EEG signal waveform of sound stimulation and analyzing the proportion of various brain wave components that continuously listen to the snow stepping sound, the psychological impact of the snow stepping sound can be objectively evaluated.
\end{abstract}

Keyword: Snow Stepping Sound, ASMR, Health, Acoustic Analysis, EEG, Brian Wave.

\section{INTRODUCTION}

Human perception of sound is very sensitive. The threshold of human hearing is $20 \mathrm{~Hz} 20 \mathrm{KHz}$. For humans, sound can not only carry the information we want to communicate for communication, but also bring sensory and psychological pleasure. This may be the reason for the birth of music. And nature is the best "musician" in the world. It creates all the sounds in this world, most of them are natural and harmonious. With the development of science and technology, people have more understanding of sound, so sound has been more and more widely used. Audible sound has been used in medicine and psychology, such as treating tinnitus, depression, controlling brain waves and so on. Infrasound and ultrasound are more commonly used in engineering. The ASMR proposed in recent years is active on various video sites, and more people are beginning to enjoy the use of certain voices to obtain sensory pleasure and psychological decompression. In fact, sound has an important impact on human health. Scientific use of natural and harmonious sound can help us live a more comfortable and beautiful life. [2] [3]
This paper will conduct an acoustic analysis and brain wave research on the psychological impact the sound of stepping on the snow to explore the application scenarios of this sound. In chapter 2, acoustic analysis of sound waveforms in the time domain and spectrum and spectrograms in the frequency domain. In chapter 3, the description of the types of snow. In chapter 4, Definition of EEG signal and brain wave type. In chapter 5, EEG is used to measure the change of EEG waveform in time domain and analysis of various brain wave components in frequency domain. In chapter 6 , conclusion. [4]

\section{TYPE OF ACCUMULATED SNOW}

Snow contains individual ice crystals, which grow when suspended in clouds, then descend, and then accumulate on the ground, where they undergo further changes. Simply put, snow is actually precipitation in the form of ice crystals, which eventually accumulate on the ground to become snow [5]. Accumulated snow is usually divided into five categories. Including powder snow, crud snow, crust snow, slush snow, Ice snow.

Powder snow: That is the snow that just fell on the ground, it is very fluffy, smooth, and very soft. Crud snow: This is the next stage of powder snow. When more people or vehicles are on the snow, the snow becomes harder after being trampled under and trampled by a car. Crust snow : When the sun and wind melt the upper layer of powder snow, and the cold freezes the melted snow again into a solid, it will crust on the surface, and the brittle shell will sit on the softer powder snow. Slush snow: Snow slush has basically begun to melt, so it has become more humid. The wet snow made the pavement muddy and rotten. Ice: Snow continues to melt into water, spread on the ground, and freeze again. Very flat and smooth. Because it is extremely slippery, it is very difficult to walk on it. This paper studies the first type of relatively thick snow that has just landed on the ground, that is, the sound of pedaling on powder snow. [6]

\section{ACOUSTIC ANALYSIS}

From the sound waveform, it can be found that the appearance and disappearance of the beat of the waveform, which is related to the recording of the person's footstep. The sound appearance period is about 1 second. That is, this person walks at a step rate of one second. when the foot stepped on the snow, the snow 
was gradually compressed, the friction between the snow became louder and louder, and the volume gradually increased. The spectrogram also shows the discontinuity of sound, which has a higher energy distribution at low frequencies, and the energy distribution is relatively flat across the entire spectrum. Higher energy appears between $50 \mathrm{~Hz}-5000 \mathrm{~Hz}$, this frequency band is the range of the fundamental frequency of human vocal cords. In order to make human voice communication barrierfree, the human ear is very sensitive to the sound in this frequency band. In other words, the high-energy sound in this band is more likely to attract people's attention. Acoustic energy gradually increases from $0 \mathrm{~Hz}$ to $150 \mathrm{~Hz}$, and peaks at $150 \mathrm{~Hz}$. Mid-range and high-frequency energy above $150 \mathrm{~Hz}$ gradually decreases, and each double frequency decreases by approximately $4 \mathrm{~dB}$, which is similar to the spectral characteristics of pink noise ( $-3 \mathrm{~dB} /$ octave). Pink noise is one of the most common noises in biological systems. This widespread sound in nature can bring people a feeling of nature, relaxation and comfort. It is a sound that people love. From the highest energy of $150 \mathrm{~Hz}$ to the lowest $16 \mathrm{Khz}$, the acoustic energy is reduced by $22 \mathrm{~dB}$. The spectrum is relatively flat, and a large amount of acoustic energy is distributed over a wide spectrum. [7] [8]

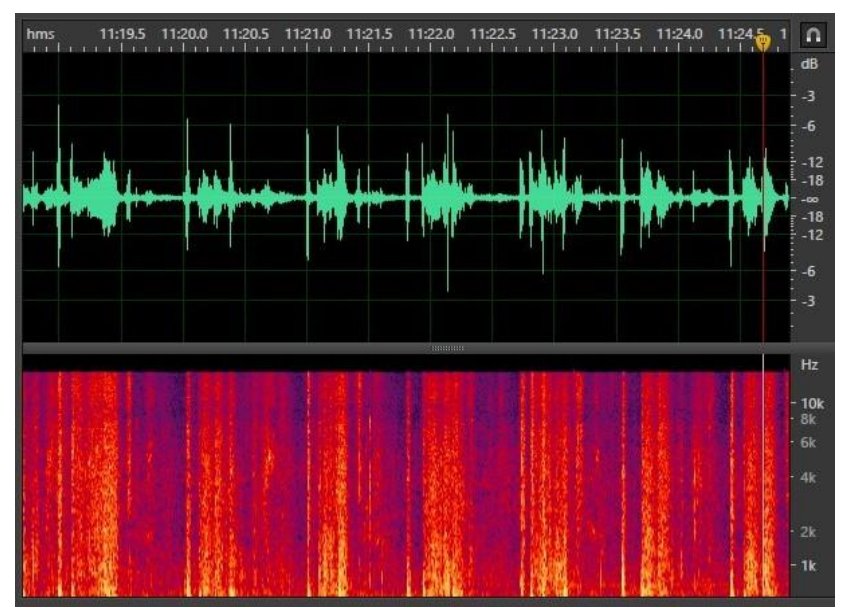

Figure 1. Waveform and spectrogram of snow stepping sound

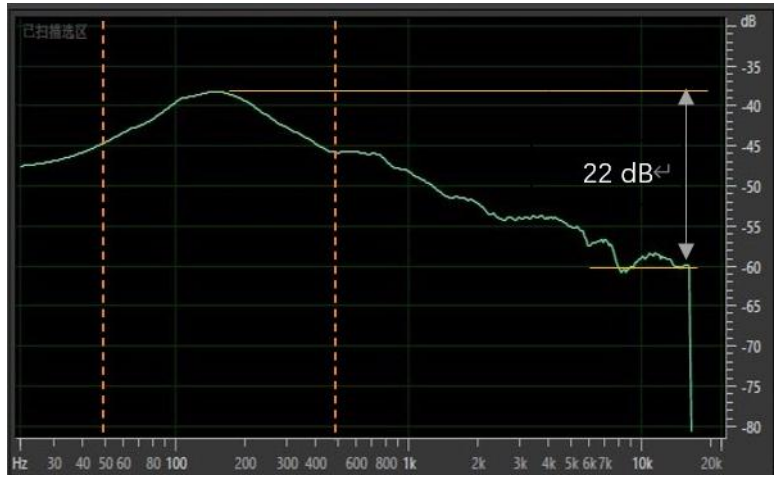

Figure 2. Spectrum analysis of Snow Stepping Sound

\section{DEFINITION OF EEG SIGNAL AND BRAIN WAVE TYPE}

Electroencephalography (EEG) is an electrophysiological monitoring method for recording electrical activity in the brain [9]. EEG signals reflect the electrical activity of brain neurons in the cerebral cortex and scalp. It is an important way to investigate the physical and psychological status of participants. The measurement method is to use non-invasive electrodes placed along the scalp. EEG measurement records voltage fluctuations caused by ion currents in brain neurons [10]. An electroencephalogram refers to a record of the brain's spontaneous power generation activity over a period of time. The horizontal axis is time and the vertical axis is voltage. Diagnostic applications usually focus on the detection of eventrelated potentials or the spectral content of EEG. Therefore, it is usually divided into time-domain analysis and frequencydomain analysis. For time-domain analysis, it is mainly to observe the degree of change of the waveform with the event of the stimulus, that is, event-related potential. For frequencydomain analysis, FFT transform the time-domain waveform to frequency domain. Is to analyze its spectral composition, the current research has distinguished various brain waves and their effects according to frequency. There are four main types of brain waves: $\delta$ wave, $\theta$ wave, $\alpha$ wave and $\beta$ wave. Beta waves are generally divided into low beta waves $(\beta \mathrm{L})$ and high beta waves $(\beta \mathrm{H})$. Table 1 is the classification of brain waves and brain function [11].

Table 1. Classification of brain waves [11]

\begin{tabular}{|c|c|l|}
\hline Types (Frequency) & Amplitude & Human brain activity \\
\hline $\begin{array}{c}\boldsymbol{\delta} \text { wave } \\
(\mathbf{1 - 3 h z})\end{array}$ & $20-200 \mu \mathrm{V}$ & $\begin{array}{l}\text { In most cases, it occurs in deep sleep. Neurons in the cortex are in a state of simultaneous relaxation, } \\
\text { which is an unconscious brain wave. }\end{array}$ \\
\hline $\begin{array}{l}\boldsymbol{\theta} \text { wave } \\
(\mathbf{4 - 7 h z )}\end{array}$ & $200-150 \mu \mathrm{V}$ & $\begin{array}{l}\text { It is a brain wave that appears between awake and sleep states, it is the performance of the central } \\
\text { nervous system's inhibition state, and is related to the learning and memory processes. }\end{array}$ \\
\hline $\begin{array}{l}\mathbf{\alpha} \text { wave } \\
(\mathbf{8 - 1 3 h z )}\end{array}$ & $\begin{array}{l}\text { It is the main brain wave that appears in a normal quiet state, indicating that in a quiet state, the } \\
\text { cerebral cortical nerve cells are in a state of relaxation and preparation for activity. The appearance of } \\
\text { a Wave can make people feel relaxed quickly. }\end{array}$ \\
\hline $\begin{array}{l}\boldsymbol{\beta} \text { wave } \\
(\mathbf{1 4 - 3 0 )}\end{array}$ & $\begin{array}{l}\text { It occurs when people perform behavioral actions and increase attention, such as thinking about } \\
\text { problems or performing intellectual activities. However, the continuous emergence of a large number } \\
\text { of } \beta \text { waves will give a lot of pressure and tension. }\end{array}$ \\
\hline
\end{tabular}




\section{EXPERIMENTS AND RESULTS}

\subsection{Experimental Method}

First record the sound source of one-minute Sound of stepping on the snow, and save it as wav format. Then start the brain wave test experiment. The subjects were 4 college students aged 20-28. The test instrument used in the experiment is a twochannel, $45 \mathrm{~Hz}$ bandwidth brain wave measuring instrument produced by Neuro Harmory S. The total sampling rate is 512 $\mathrm{Hz}$, and the single channel sampling frequency is $256 \mathrm{~Hz}$. The experiment site is located in a quiet laboratory.

In order to record the difference between the test subject's EEG data in the quiet mode and the stimulation mode of the snow stepping sound. First, recorded the brain wave data for one minute without applying any sound stimulation to the test subject, then added the snow stepping sound stimulation to record for one minute. The recording time of the brain wave data of one test subject lasted for two minutes. During the test time, test subjects need to keep quiet and close their eyes.[12]

The experimental data can be divided into three parts. The first part is the EEG data when it is quiet. The second part is to intercept the EEG data from the quiet state to the time of receiving the sound stimulation, and the third part is the EEG data that continuously receives the sound stimulation. The FFT transformation of the first and third parts can analyze the difference of various brain wave contents in the two states, and the temporal waveform changes during the sound stimulation can be observed in the time-domain EEG waveform of the second part. [13]

\subsection{Experimental Results}

Figure 3 is the EEG waveform of intercepting the sound stimulation. It shows that brain wave waveform of 4 seconds, included the first 2 seconds is the brain wave when it is quiet, and the second 2 seconds is the brain wave that listens snow stepping sound. When a sound stimulus was added at the second seconds (red line), a sudden change in the waveform is detected at approximately $2.15 \mathrm{~s}$ (green line). The amplitude of the waveform suddenly increases and then decreases, and then returns to normal. This means that the brain has received sound stimuli and triggers event-related potentials. The brain responded to the sound. [14]

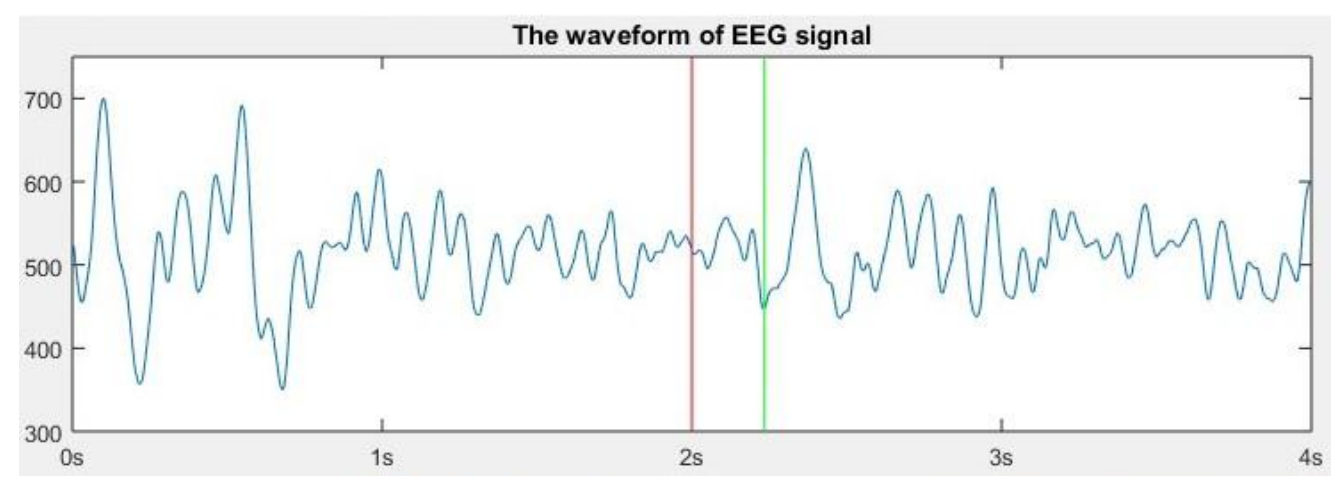

Figure 3. EEG waveform during sound stimulation transition

Continue to intercept the EEG data of $0-1 \mathrm{~s}$ and $3-4 \mathrm{~s}$. This is the brainwave data 1 second before and 1 second after the sound stimulation, respectively. After obtaining auto-correlation and FFT transformation on these two pieces of data, their short time power spectrum density is obtained.

Following is the short time power spectral density function.

$$
R_{n}(k)=\sum_{m=-\infty}^{\infty} x(m) W(n-m) x(m+k) W(n-m+k)
$$

We can define:

$$
\begin{aligned}
h_{k} & =W(n) W(n+k) ; \\
R_{n}(k) & =\sum_{m=-\infty}^{\infty} x(m) x(m-k) h_{k}(n-m) \\
& =[x(m) x(m-k)] * h_{k} ;
\end{aligned}
$$

Therefore, the short-term autocorrelation function can be regarded as the output of the sequence $x(m) x(m-k)$ through the $h_{k}$ filter. Where the $\mathrm{W}(\mathrm{n})$ is FFT windows that is a hamming window with 256 point.
And, autocorrelation function and power spectral density function are Fourier transforms of each other.

$$
\begin{gathered}
F_{n}(K) \stackrel{D F T}{\Longleftrightarrow} R_{n}(k) ; \\
F_{n}(K)=\sum_{n=0}^{N-1} R_{n}(k) e^{-j \frac{2 \pi}{N} K n} .
\end{gathered}
$$

As shown in Figure 4, the blue line is the EEG signal power spectral density curve at rest. The red line is the power spectral density curve of the EEG signal when there is sound. The three green lines divide the frequency axis into 4 brainwave regions. They are $\delta$ wave, $\theta$ wave, $\alpha$ wave and $\beta$ wave. For the delta wave band and the alpha wave band, the brain wave content in the quiet state and the sound state is similar. Under the sound state, the brain wave content in the $\theta$ wave band is significantly reduced, but the brain wave content in the $\beta$ wave band is increased. When listening to the sound of treading snow, the $\theta$ wave decreases and the $\beta$ wave increases, which shows that the snow treading sound can relieve fatigue, and improve people's attention and mobility. And because there is no obvious change in the $\alpha$ wave, the sound of treading snow does not bring tension and stress, and it can still keep people calm and relaxed. [15] 


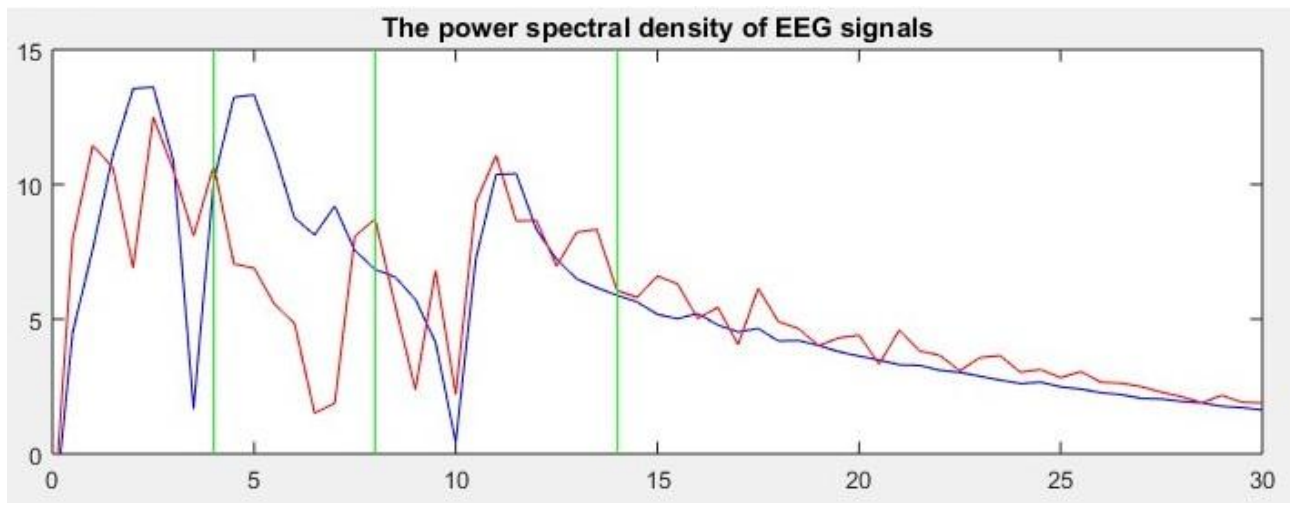

Figure 4. EEG signal power spectral density curve when quiet (blue) and EEG signal power spectral density curve when listening to snow stepping sound (red)

In summary, the analysis of one-second transient brain wave components before and after stimulation is explained. Figure 4 is a bar graph of the percentage of brainwaves of four test subjects one minute before and after listening to the treading sound. It compares the analysis of various brain wave contents before and after listening to the treading sound. The FFT window length is 256 points ( 1 second). The 60 values of each brain wave measured within one minute are averaged, and the percentage is compared. As shown in the figure, the four test subjects had the same change trend in the percentage of brain waves when listening to the sound of treading snow compared to when they were quiet. That is, the percentages of $\delta$ and $\theta$ waves decrease, while the percentages of $\alpha$ and $\beta$ waves increase. Listening to the sound of treading snow can indeed keep people's brains awake, which can effectively improve people's attention and reaction ability, thereby improving work efficiency. [16]

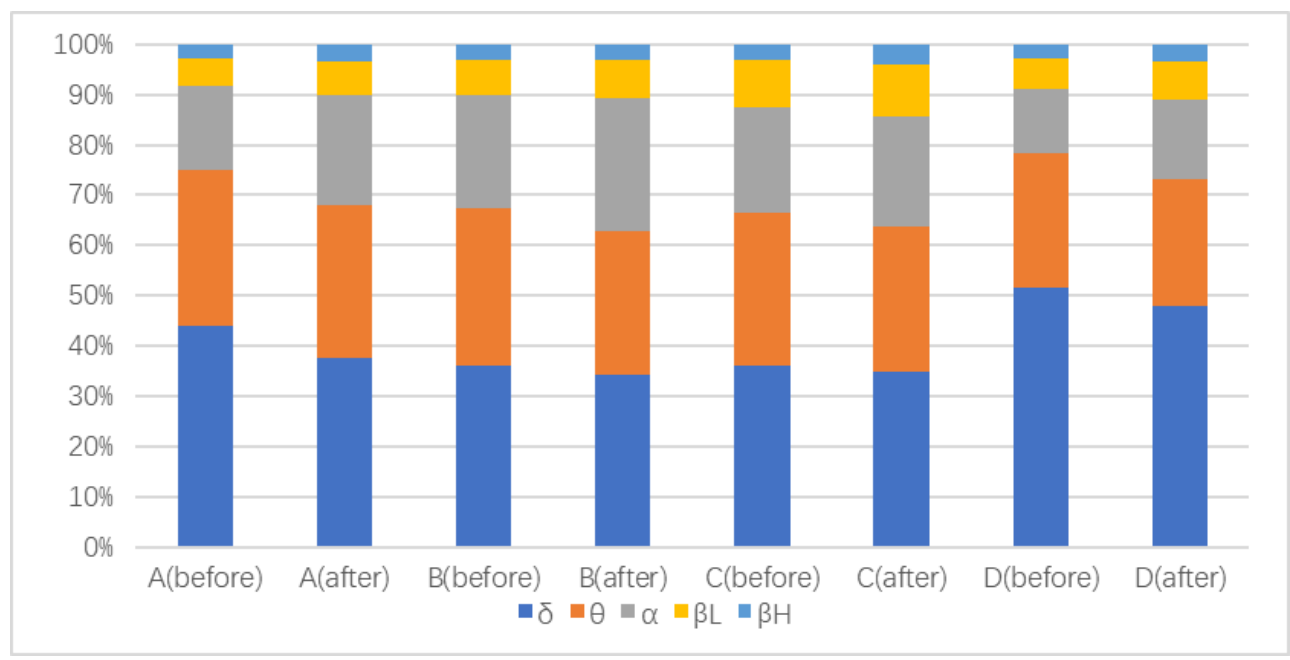

Figure 5. The percentage of brain waves before and after the four test subjects listened to snow stepping sound

\section{CONCLUSION}

Sound can stimulate the brain, thereby affecting people's mental state and mood changes. So, people use this principle to obtain spiritual feelings through sound stimulation. In fact, the stimulation of the brain by sound may even affect human cognitive ability and action ability.[17]

The sound of treading snow is one of the most popular natural sounds. In time domain, the sound shows discontinuity and beat. In the frequency domain, it is similar to many natural sounds, with a wide spectrum energy distribution, and the overall energy distribution characteristics are similar to pink noise. However, there is a high-energy distribution in the $50 \mathrm{~Hz}$ to
$500 \mathrm{~Hz}$ frequency band, which is the range of the fundamental frequency of human vocal cords. The high-energy condensed sound in this frequency band can more attract the attention of the human hearing system.

Brain wave analysis test results showed that event-related potentials were observed at the time point of sound stimulation. And the ratio of brain wave content before and after listening to the sound is different. Listening to the snow stepping sound can induce more $\alpha$ waves and $\beta$ waves, while suppressing $\delta$ waves and $\theta$ waves. Therefore, listening to the sound of treading snow can relieve fatigue and stress, and improve people's attention and alertness. With this feature, people can 
be well used in various application scenarios. For example, it is used in an office environment, which can alleviate the fatigue of people working for a long time and improve work efficiency. Playing this sound while driving a car can also avoid accidents caused by driver fatigue driving. In future research, we should continue to study the effect of sound playback time and volume on this excitation effect.

\section{REFERENCE}

[1] Ik-Soo Ahn, Bong-young kim, Myung-jin Bae. "A study on the human sensation of the reed wind sound in ASMR". International Journal of Engineering Research and Technology Volume 12, Issue 9, 2019, Pages 14941499.

[2] Kyoung-su Yeo, Myungsook Kim, Myung-Jin Bae. "Acoustic characteristics of the forest sounds inducing sleep. Information (Japan) Volume 18, Issue 10, October 2015, Pages 4407-4412.

[3] Bong-Young Kim, Zhixing Tian, Myung-Jin Bae. "A study on the causes of revenge psychology of Klaxon sound". International Journal of Engineering Research and Technology Volume 12, Issue 12, 2019, Pages 29472952.

[4] Jens Kreitewolf, Jörg Lewald, Stephan Getzmann, "Effect of attention on cortical processing of sound motion: An EEG study", NeuroImage, Volume 54, Issue 3, 2011, Pages 2340-2349.

[5] Wikipedia. [https://en.wikipedia.org/wiki/Snow].

"snow".

[6] SNOW GUIDE. "5 Types of Snow and How They Function". [http://snow.guide/snow-types/].

[7] Zhixing Tian, Bong-Young Kim, Myung-Jin Bae. "Study on the acoustic characteristics of Sunwapta Falls". International Journal of Engineering Research and Technology, Volume 13, Issue 5, 2020, Pages 962-966.

[8] Zhixing Tian, Bong-Young Kim, Myung-Jin Bae. "Study on acoustic analysis of Cleveland Dam waterfall sound".
International Journal of Engineering Research and Technology 13(6), To be published.

[9] Wikipedia. "Electroencephalography". [https://en.wikipedia.org/wiki/Electroencephalography].

[10] Y. Sun, N. Ye, X. Wang and X. Xu, "The Research of EEG Analysis Methods Based on Sounds of Different Frequency," 2007 IEEE/ICME International Conference on Complex Medical Engineering, Beijing, 2007, pp. 1746-1751.

[11] Personal 1ibrary. "The four brains of human". [http://www.360doc.com/content/15/0714/17/15991787_ 484900161.shtml].

[12] Guo-Qing Di, Meng-Chi Fan,Qin-Hao Lin . "An experimental study on EEG characteristics induced by intermittent pure tone stimuli at different frequencies". Applied Acoustics, Volume 141, 2018, Pages 46-53.

[13] Ik-Soo Ahn, Bong-Young Kim, Kwang-Bock You, Myung-Jin Bae "A Study on the Characteristics of an EEG Based on a Singing Bowl's Sound Frequency", Studies in Computational Intelligence Volume 789, 2019, Pages 233-243.

[14] Ahn, I.-S., Bae, M.-J., Bae, S.-G. "A study on the sound of dadmi according to a type of cloth using an acoustics analysis". (2018) International Journal of Engineering Research and Technology, 11 (7), pp. 1003-1014.

[15] Sturm I, Dähne S, Blankertz B, Curio G (2015) MultiVariate EEG Analysis as a Novel Tool to Examine Brain Responses to Naturalistic Music Stimuli. PLoS ONE 10(10): e0141281. doi:10.1371/ journal. pone.0141281.

[16] Ahn, I.-S., Kim, B.-Y., You, K.-B., Bae, M.-J.“AA Study on the Characteristics of an EEG Based on a Singing Bowl's Sound Frequency".(2019) Studies in Computational Intelligence, 789, pp. 233-243.

[17] Rausch, V. H. (2014). White noise improves learning by modulating activity in dopaminergic midbrain regions and right superior temporal sulcus. Journal of cognitive neuroscience, 1469-1480 